\title{
Helium abundances in HgMn and normal stars
}

\author{
M.M. Dworetsky \\ Department of Physics and Astronomy, University College London, Gower Street, London \\ WC1E 6BT, UK \\ email: mmd@star.ucl.ac.uk
}

\begin{abstract}
The parameter-free model of diffusion in the atmospheres of HgMn stars (Michaud 1986, Michaud et al. 1979) predicts that helium should sink below the He II ionization zone that the diffusion of other elements may take place, and that all HgMn stars should have deficits of helium in their photospheres, with a minimum deficit of 0.3 dex. In this study, the Smith \& Dworetsky (1993) sample of HgMn stars and normal comparison stars is examined, and the helium abundances determined by spectrum synthesis using échelle spectra taken at Lick Observatory and the AAT. The prediction is confirmed. All HgMn stars are deficient in He by as much as 1.5 dex. Also, two HgMn stars, HR 7361 and HR 7664, show clear evidence of helium stratification.
\end{abstract}

Keywords. Stars: abundances, stars: chemically peculiar

\section{Introduction}

Abundances were determined for $25 \mathrm{HgMn}$ stars and 12 normal and superficially normal stars of similar $T_{\text {eff }}$, using an LTE analysis. It is well-known that the effects of non-LTE can safely be ignored in the relevant temperature range. The analysis was performed for two He I lines, $\lambda 4026.2$ and $\lambda 4471.5$. The line profile tables of Barnard et al. $(1969,1974,1975)$ and Shamey (1969) were used. The abundances and estimated errors were obtained here by trial and error fits by eye to the observations. The unweighted mean for the normal stars is $\log N(\mathrm{He}) / N(\mathrm{H})=10.98 \pm 0.05$, in excellent agreement with the standard value 10.99 (Grevesse et al. 1996). (In this paper all abundances are given on the scale $\log N(\mathrm{H})=12.00$.) It is found that all HgMn stars have underabundances, ranging from factors of $0.3 \mathrm{dex}$ at low $T_{\text {eff }}$ to $1.5 \mathrm{dex}$ at high $T_{\text {eff }}$. These observations provide direct support for the parameter-free model.

\section{Observations}

The programme stars are listed in Table 1. Most of the observations were made with the Hamilton Échelle Spectrograph at the Lick Observatory, with FWHM resolution $R=46500$. Two southern stars ( $\xi$ Oct and $\beta \mathrm{Scl}$ ) were observed with the UCLES on the Anglo-Australian Telescope with nearly identical resolution. Further details of the observations and reductions, and treatment of binary stars, are the same as in Jomaron et al. (1999).

\section{Abundance of $\mathrm{He}$}

Auer \& Mihalas (1973) showed that the He I lines used in this work can be wellapproximated by LTE models for B stars in the temperature range below $15000 \mathrm{~K}$. The 
Table 1. Stellar parameters and helium abundances for the programme stars.

\begin{tabular}{|c|c|c|c|c|c|c|c|c|}
\hline Star & HD & $\begin{array}{l}T_{\text {eff }} \\
(\mathrm{K})\end{array}$ & $\begin{array}{l}\log g \\
(\operatorname{cgs})\end{array}$ & $\begin{array}{c}\xi \\
\mathrm{km} \mathrm{s}^{-1}\end{array}$ & $\begin{array}{c}v \sin i \\
\mathrm{~km} \mathrm{~s}^{-1}\end{array}$ & $\begin{array}{c}\log N(\mathrm{He}) \\
\lambda 4471\end{array}$ & $\begin{array}{c}\log N(\mathrm{He}) \\
\lambda 4026\end{array}$ & $\begin{array}{c}\log N(\mathrm{He}) \\
\text { mean }\end{array}$ \\
\hline \multicolumn{9}{|c|}{ Normal Stars } \\
\hline$\nu$ Cap & 193432 & 10300 & 3.90 & 1.6 & 27 & $10.97 \pm 0.05$ & $10.70 \pm 0.10$ & $10.92 \pm 0.05$ \\
\hline$\alpha \operatorname{Lyr}$ & 172167 & 9450 & 4.00 & 2.0 & 24 & $10.99 \pm 0.05$ & $10.99 \pm 0.10$ & $10.99 \pm 0.05$ \\
\hline HR 7098 & 174567 & 10200 & 3.55 & 1.0 & 11 & $10.90 \pm 0.05$ & $10.50 \pm 0.05$ & $10.70 \pm 0.04$ \\
\hline$\zeta$ Dra & 155763 & 12900 & 3.90 & 2.5: & 34 & $10.99 \pm 0.05$ & $10.99 \pm 0.05$ & $10.99 \pm 0.04$ \\
\hline $134 \mathrm{Tau}$ & 38899 & 10850 & 4.10 & 1.6 & 30 & $11.05 \pm 0.05$ & $10.99 \pm 0.10$ & $11.04 \pm 0.05$ \\
\hline$\xi$ Oct & 215573 & 14050 & 3.85 & $0.5:$ & 5 & $10.99 \pm 0.10$ & $10.99 \pm 0.10$ & $10.99 \pm 0.07$ \\
\hline$\tau$ Her & 147394 & 15000 & 3.95 & 0.0 & 32 & $10.95 \pm 0.05$ & $10.92 \pm 0.10$ & $10.94 \pm 0.05$ \\
\hline $21 \mathrm{Aql}$ & 179761 & 13000 & 3.50 & 0.2 & 17 & $11.10 \pm 0.05$ & $11.00 \pm 0.10$ & $11.08 \pm 0.05$ \\
\hline$\pi$ Cet & 17081 & 13250 & 3.80 & 0.0 & 25 & $11.20 \pm 0.05$ & $10.99 \pm 0.10$ & $11.16 \pm 0.05$ \\
\hline
\end{tabular}

Superficially Normal Stars

\begin{tabular}{lllllllll}
\hline 21 Peg & 209459 & 10450 & 3.50 & 0.5 & 4 & $10.90 \pm 0.05$ & $10.85 \pm 0.05$ & $10.88 \pm 0.04$ \\
HR 7878 & 196426 & 13050 & 3.85 & $1.0:$ & 6 & $10.99 \pm 0.05$ & $10.85 \pm 0.10$ & $10.96 \pm 0.05$ \\
HR 7338 & 181470 & 10250 & 3.75 & 0.5 & 3 & $10.80 \pm 0.10$ & $10.65 \pm 0.10$ & $10.73 \pm 0.07$ \\
\hline
\end{tabular}

\begin{tabular}{lrllllrrr}
\hline & \multicolumn{7}{c}{ HgMn Stars } \\
\hline$\beta$ Scl & 221507 & 12400 & 3.90 & $0.0:$ & 27 & $9.60 \pm 0.10$ & $9.80 \pm 0.10$ & $9.70 \pm 0.07$ \\
36 Lyn & 79158 & 13700 & 3.65 & $2.0:$ & 49 & $9.90 \pm 0.10$ & $9.60 \pm 0.10$ & $9.75 \pm 0.07$ \\
$v$ Her & 144206 & 12000 & 3.80 & 0.6 & 11 & $10.20 \pm 0.05$ & $10.20 \pm 0.05$ & $10.20 \pm 0.04$ \\
HR 7361 & 182308 & 13650 & 3.55 & 0.0 & 9 & $9.5 \rightarrow 9.8$ & $9.5 \rightarrow 9.8$ & $9.65:$ \\
28 Her & 149121 & 11000 & 3.80 & 0.0 & 8 & $9.75 \pm 0.10$ & $9.90 \pm 0.10$ & $9.83 \pm 0.07$ \\
HR 7143 & 175640 & 12100 & 4.00 & 1.0 & 2 & $10.12 \pm 0.05$ & $10.30 \pm 0.05$ & $10.21 \pm 0.04$ \\
46 Aql & 186122 & 13000 & 3.65 & 0.0 & 1 & $9.25 \pm 0.10$ & $9.45 \pm 0.10$ & $9.35 \pm 0.07$ \\
HR 7775 & 193452 & 10800 & 3.95 & 0.0 & 1 & $10.0 \pm 0.30$ & $9.50 \pm 20$ & $9.65 \pm 0.17$ \\
$\kappa$ Cnc & 78316 & 13500 & 3.80 & 0.0 & 6 & $9.85 \pm 0.15$ & $9.85 \pm 0.10$ & $9.85 \pm 0.08$ \\
53 Tau & 27295 & 12000 & 4.25 & 0.0 & 5 & $9.90 \pm 0.05$ & $10.20 \pm 0.10$ & $9.96 \pm 0.05$ \\
HR 7664 & 190229 & 13200 & 3.60 & 0.8 & 8 & $9.2 \rightarrow 9.7$ & $9.4 \rightarrow 9.8$ & $9.52:$ \\
$\phi$ Her & 145389 & 11650 & 4.00 & 0.4 & 10 & $10.20 \pm 0.05$ & $10.38 \pm 0.05$ & $10.29 \pm 0.04$ \\
$\phi$ Phe & 11753 & 10700 & 3.80 & $0.5:$ & 13 & $9.80 \pm 0.15$ & $10.00 \pm 0.10$ & $9.94 \pm 0.05$ \\
$\nu$ Cnc & 77350 & 10400 & 3.60 & 0.1 & 13 & $10.30 \pm 0.05$ & $10.10 \pm 0.10$ & $10.26 \pm 0.05$ \\
HR 2844 & 58661 & 13460 & 3.80 & $0.5:$ & 30 & $10.00 \pm 0.10$ & $10.00 \pm 0.10$ & $10.00 \pm 0.07$ \\
33 Gem & 49606 & 14400 & 3.85 & $0.5:$ & 22 & $9.50 \pm 0.10$ & $9.75 \pm 0.15$ & $9.58 \pm 0.08$ \\
$\mu$ Lep & 33904 & 12800 & 3.85 & 0.0 & 18 & $9.65 \pm 0.10$ & $10.05 \pm 0.05$ & $9.97 \pm 0.05$ \\
HR 2676 & 53929 & 14050 & 3.60 & $1.0:$ & 25 & $9.30 \pm 0.15$ & $9.70 \pm 0.30$ & $9.38 \pm 0.13$ \\
87 Psc & 7374 & 13150 & 4.00 & 1.5 & 21 & $10.25 \pm 0.10$ & $10.30 \pm 0.10$ & $10.27 \pm 0.07$ \\
HR 6997 & 172044 & 14500 & 3.90 & 1.5 & 34 & $9.72 \pm 0.05$ & $9.85 \pm 0.05$ & $9.79 \pm 0.04$ \\
HR 4072 & 89822 & 10650 & 3.95 & 1.0 & 3.2 & $10.40 \pm 0.10$ & $10.40 \pm 0.10$ & $10.40 \pm 0.07$ \\
$\chi$ Lup $^{a}$ & 141556 & 10650 & 4.00 & 0.0 & 2.0 & $10.76 \pm 0.04$ & $10.70 \pm 0.07$ & $10.74 \pm 0.02$ \\
$\iota$ CrB & 143807 & 11000 & 4.00 & 0.2 & 1.0 & $10.35 \pm 0.10$ & $10.42 \pm 0.05$ & $10.36 \pm 0.04$ \\
112 Her & 174933 & 13100 & 4.10 & $0.0:$ & 6 & $9.58 \pm 0.05$ & $9.65 \pm 0.10$ & $9.60 \pm 0.04$ \\
HR 1800 $^{a}$ & 35548 & 11050 & 3.80 & 0.5 & 3 & $10.55 \pm 0.05$ & $10.45 \pm 0.05$ & $10.50 \pm 0.04$ \\
\hline Bring $^{a}$ & & & & & & & &
\end{tabular}

${ }^{a}$ Binaries with two spectra (parameters refer to the primary). Explicit allowances for dilution effects have been made in this work.

${ }^{b}$ See text and Figs. 5 and 6 for a discussion of HR 7664 and HR 7361.

spectrum synthesis code UCLSYN was used to calculate the abundance of He for 9 normal stars, 3 superficially-normal stars, and $25 \mathrm{HgMn}$ stars. The results are shown in Table 1 and Fig 1. It is clear from this figure that all HgMn stars in this sample are deficient in $\mathrm{He}$ by 0.3 dex or more (usually much more). It also appears that the hotter HgMn stars have stronger He deficits than the cooler HgMn stars. Dividing them into 12 cooler and 13 
hotter HgMn stars yields $\log N(\mathrm{He})=10.20 \pm 0.09$ for the cooler group and $\log N(\mathrm{He})=$ $9.72 \pm 0.07$ for the hotter group. The statistical difference is highly significant.

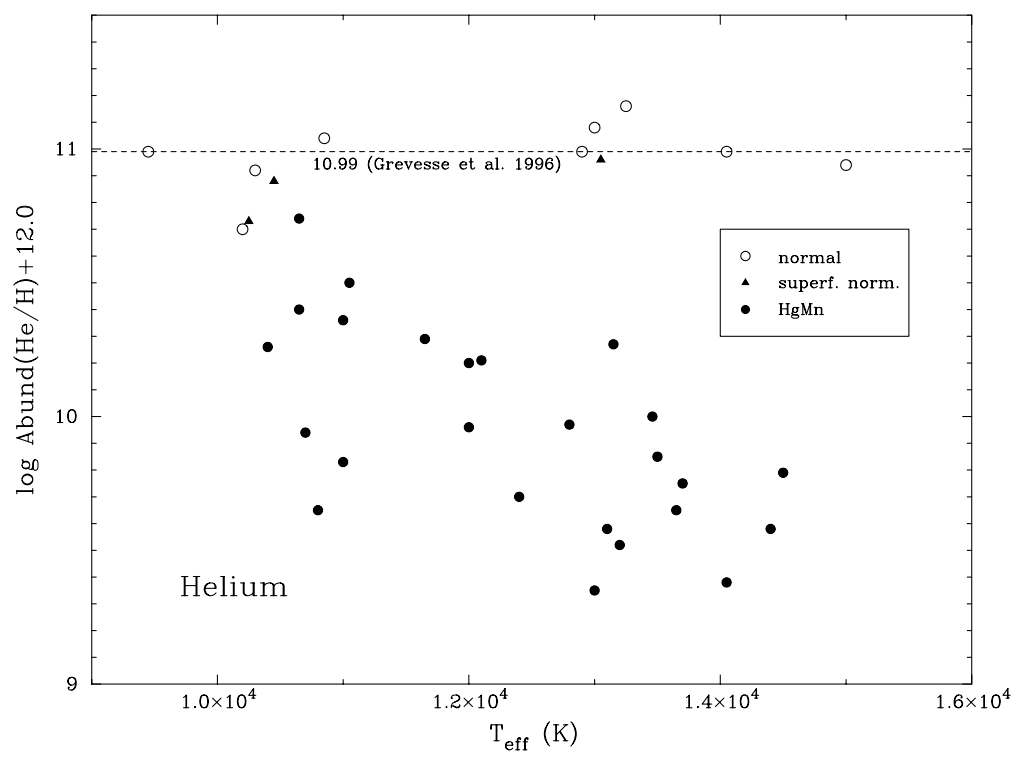

Figure 1. Helium abundances in normal stars, superficially normal stars, and HgMn stars. Points are averages of $\lambda 4026$ and $\lambda 4471$ profile fits. Typical errors (not shown) are \pm 0.06 dex. There is more depletion of He in the hotter HgMn stars than in the cooler group. In two cases, where stratification of He is suspected, the values are the means of the best fits to line centres and wings (see text).

Examples of fits to normal and HgMn stars are shown in Figs 2, 3 and 4.

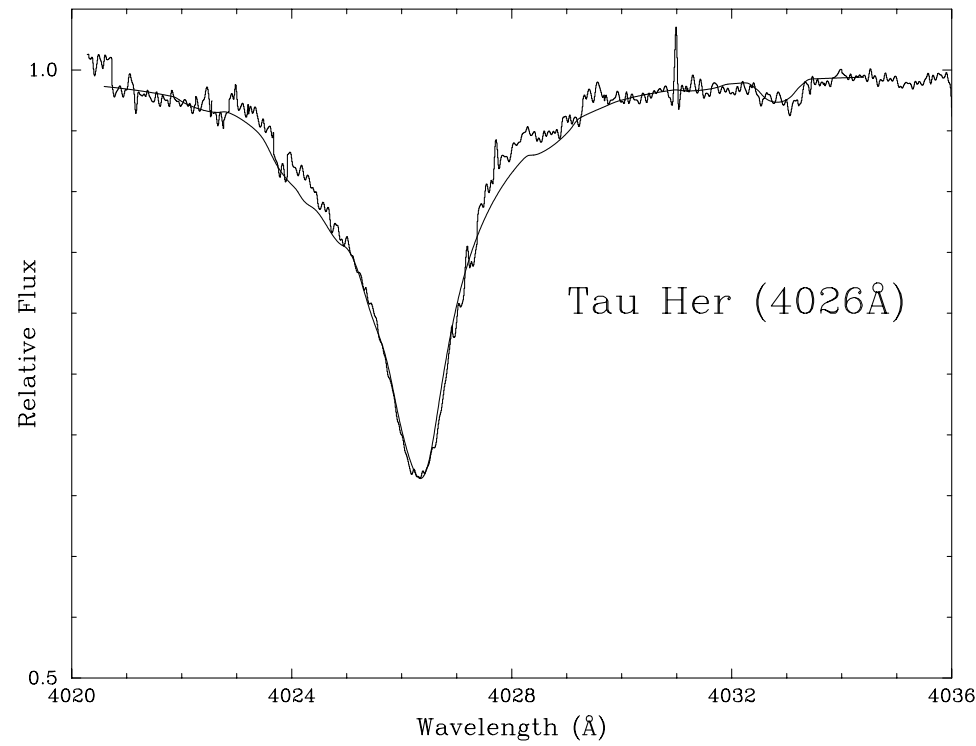

Figure 2. The line profile fit for $\tau$ Her, a normal B5 IV star with $T_{\text {eff }}=15000 \mathrm{~K}, \log g=3.95$, and derived He abundance 10.94. 


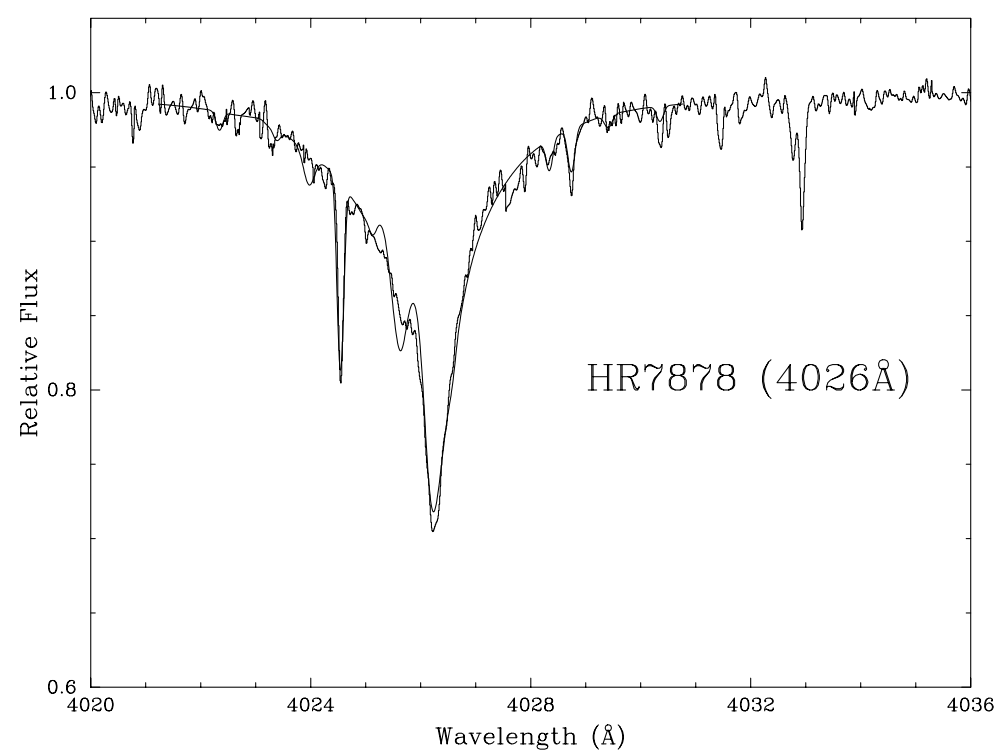

Figure 3. The line profile fit for HR 7878 (B8 IIIp), a superficially normal star with $T_{\text {eff }}=$ $13050, \log g=3.85$, with He abundance 10.85 for $\lambda 4026$.

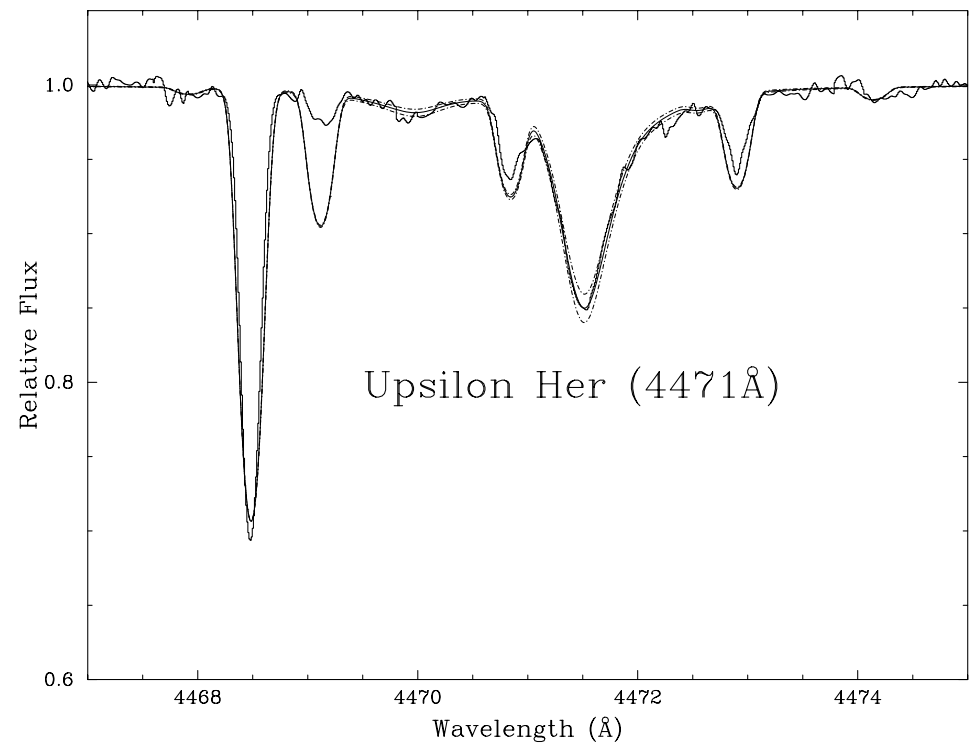

Figure 4. Best fit for the HgMn star $v$ Her $\lambda 4471, T_{\text {eff }}=12000 \mathrm{~K}, \log g=3.80$, with He abundance 10.20. For illustration, the dash-dot lines indicate the profiles for changes of abundance of \pm 0.08 dex, larger than the error estimate in this work.

Typical errors of single determinations by fitting one line were $\pm 0.05-0.10$ dex, and consistency between $\lambda 4026$ and $\lambda 4471$ was of similar quality. Blends which occurred within the profile were modelled. 

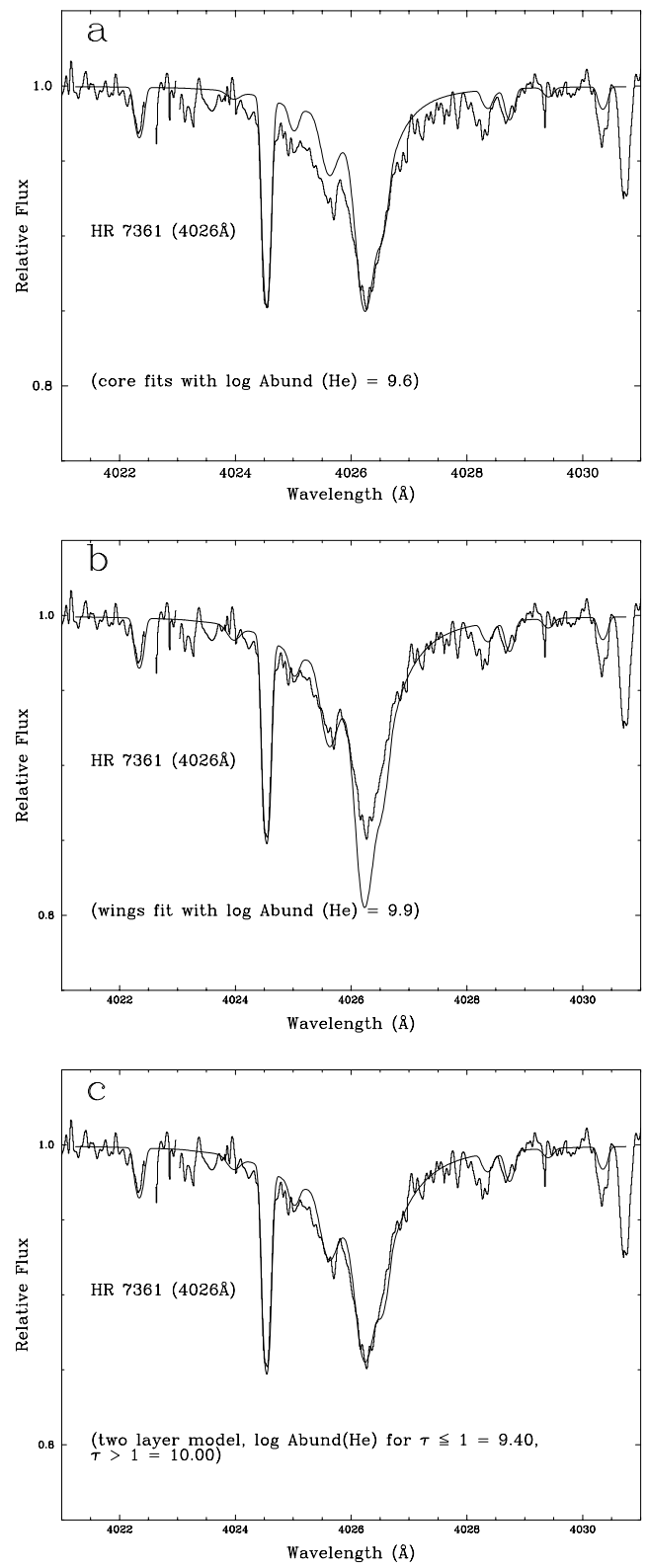

Figure 5. The centre or core (a) of $\lambda 4026 \mathrm{He}$ I fits a very low He abundance in the HgMn star HR 7361. The wings can only be fit with a higher abundance (b), but then the core is a bad fit. A model with a higher abundance of He below $\tau=1$ (c) fits both core and wings reasonably well. This indicates that He is stratified, with more depletion in the upper photosphere than in the deeper photosphere.

\section{Depth dependent He abundances}

In nearly all cases, the profile fits to wings and centres of the two lines were fully consistent, indicating that the modelling assumption of uniform fractional abundance of He with depth was a good one. However, two HgMn stars, HR 7361 (Fig. 5) and HR 7664 (Fig. 6) could not be fit to one abundance. In both cases the centre gave a good fit only 

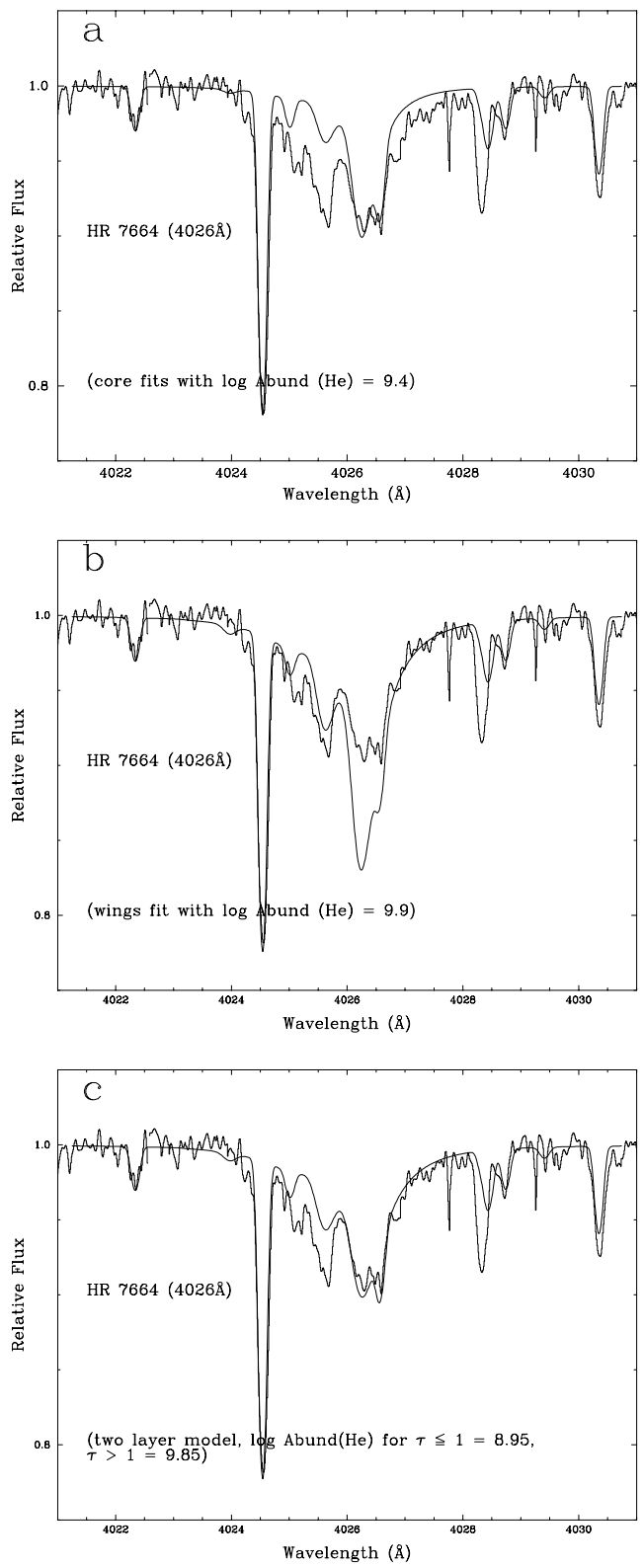

Figure 6. The HgMn star HR 7664 has similar fitting problems to HR 7361 shown in Fig. 5. The core (a) of $\lambda 4026 \mathrm{He}$ I fits a low He log abundance, 9.4. The wings require a higher value 9.9 (b), but then the core is a bad fit. A model with a higher abundance of He below $\tau=1$ (c) fits both core and wings reasonably well. Again, this shows that He is stratified, with more depletion in the upper photosphere than in deeper layers.

for abundances about 0.3-0.5 dex lower than the fit in the wings. A model of the $\mathrm{He}$ abundance in these two stars with an enhanced abundance below $\log \tau=1.0$ produced much more satisfactory fits. Although the solutions in Figs. $5 \mathrm{c}$ and $6 \mathrm{c}$ are not unique, as the actual depth distribution is probably more complicated, they are indicative of the fact that He must be considerably more depleted in the higher photosphere than in 
deeper layers, although it is also depleted there as well. It appears that He has left a clear trace of its downwards diffusion through the He II convection zone in these two cases.

\section{Acknowledgements}

I am grateful to Prof. J. S. Miller, Director of Lick Observatory for observing time for this work, and to Mr. D. Stansall, MSci, for his excellent efforts on He during a supervised undergraduate research project at University College London.

\section{References}

Auer L.H., Mihalas, D. 1973, ApJS 25, 433

Barnard, A.J., Cooper, J., Shamey, L.J. 1969, A\& A 1, 28

Barnard, A.J., Cooper, J., Smith, E.W. 1974, JQSRT 14, 1025

Barnard, A.J., Cooper, J., Smith, E.W. 1975, JQSRT 15, 429

Grevesse, N., Noels, A., Sauval, A.N. 1996, ASP Conf Series 99, 117

Jomaron, C.M., Dworetsky, M.M., Allen, C.S. 1999, MNRAS 303, 555

Michaud, G. 1986, in: Cowley, C.R., Dworetsky, M.M., Mégessier, C., (eds), Upper Main Sequence Stars with Anomalous Abundances, IAU Coll. 90, (D. Reidel, Dordrecht), p. 459

Michaud, G., Martel, A., Montmerle, T., Cox, A.N., Magee, N.H., Hodson, S.W. 1979, ApJ 234, 206

Shamey, L. J. 1969, PhD thesis, University of Colorado

Smith, K.C., Dworetsky, M.M. 1993, A\&A 274, 335. 